\title{
TWO RESULTS RELATED TO A QUESTION OF HINKKANEN
}

\author{
J.K. LANGLEY
}

\begin{abstract}
We prove two results concerning meromorphic functions $f$ and $g$ of finite order such that $f^{(j)}$ and $g^{(j)}$ have the same zeros and poles, for $j=0,1,2$.
\end{abstract}

\section{Introduction}

In this paper we use standard notation associated with value-sharing problems problems [2, et. al.]. The meromorphic functions $f$ and $g$ share the value $a$ IM (ignoring multiplicities) if every $a$-point of $f$ is an $a$-point of $g$, and viceversa, and $\mathbf{C M}$ (counting multiplicities) if, in addition, the multiplicities coincide. The following problem was proposed by A. Hinkkanen [1].

Suppose that $f$ and $g$ are meromorphic in the plane such that $f^{(j)}$ and $g^{(j)}$ share 0 and $\infty \mathbf{C M}$, for $0 \leqq j \leqq n$. How large need $n$ be in order to determine some relationship between $f$ and $g$ ? In fact, for entire $f$ and $g$ of finite order, this problem had already been considered by Gundersen [2]. The first published results for meromorphic $f$ and $g$ seem to be those in the thesis of Köhler [4, $5]$, who proved (Satz 4.9 ) that $n=6$ suffices to conclude that one of the following holds :

$$
\begin{gathered}
f / g \text { is constant ; } f(z)=e^{a z+b}, \quad g(z)=e^{c z+d} ; \\
f(z)=a /\left(1-b e^{h(z)}\right), \quad g(z)=c /\left(e^{-h(z)}-b\right) ; \\
f(z)=a\left(1-b e^{c z}\right), \quad g(z)=d\left(e^{-c z}-b\right) ; \quad a, b, c, d \in C, h \text { entire. }
\end{gathered}
$$

Moreover (Satz 3.1), if $f$ and $g$ have finite order, then one of the conclusions (1.1) holds if $f^{(j)}$ and $g^{(j)}$ share 0 and $\infty \mathbf{C M}$, for $j=0,1, k$, for some $k \geqq 2$. Further results were proved by Tohge [8] for functions of hyper-order less than 2 such that $f^{(j)} / g^{(j)}$ is entire and zero-free for $j=0,1,2$. We shall prove the following theorem.

THEOREM 1. (i) Suppose that $f$ and $g$ are functions meromorphic of finite

A.M.S. Classification 30D35.

Received September 19, 1994 ; revised April 4, 1995. 
order in the plane, with $g$ non-constant, such that $f / g$ and $f^{\prime} / g^{\prime}$ have only finitely many zeros and poles. Then one of the following holds:

(a) $f / g$ is constant.

(b) $f^{\prime} / f$ and $g^{\prime} / g$ are both rational.

(c) There exist a rational function $S$ and a non-constant polynomial $Q$ and constants $d$, such thet

$$
f=d_{1}\left(S e^{Q}-1\right)^{d_{2}}, \quad g=d_{3}\left(1-S^{-1} e^{-Q}\right)^{d_{2}} .
$$

(d) There exist non-constant polynomials $Q$ and $P_{1}$ such that

$$
f^{\prime} / f=\frac{P_{1}(Q) Q^{\prime}}{1-e^{-Q}}, \quad g^{\prime} / g=\frac{P_{1}(Q) Q^{\prime}}{e^{Q}-1} .
$$

(ii) If, in addition, $f^{\prime \prime} / g^{\prime \prime}$ has only finitely many zeros and poles then we have either (a), (b), or (c), with $d_{2}= \pm 1$.

Gundersen [2] proved a result similar to (i), for $f$ and $g$ entire of finite order, such that $f / g$ and $f^{\prime} / g^{\prime}$ have no zeros or poles. Our approach is different to those of $[2,4,8]$, being based not on Nevanlinna theory but rather on a simple application of the following lemma, proved in [7] using a modified form of the forward-difference argument of $[9$, p. 52].

LEMMA A. Suppose that $h(z)$ is analytic in the closed half-plane $\operatorname{Re}(z) \geqq 0$, with $\log ^{+}|h(z)| \leqq O\left(1+\log ^{+}|z|\right)$ there, and suppose that for all sufficiently large positive integers $n$, we have $h(n) \in Z$. Then $h$ is a polynomial.

Theorem 1 serves partly as an auxiliary result for the proof of the following theorem, in which we relax the assumption that $f$ and $g$ share $\infty \mathbf{C M}$.

THEOREM 2. Suppose that $f$ and $g$ are functions meromorphic of finite order in the plane such that $f^{(j)}$ and $g^{(j)}$ share the value $0 \boldsymbol{C M}$, for $j=0,1,2$, and such that $f$ and $g$ share the value $\infty \boldsymbol{I M}$. Then one of the following holds.

(i) $f / g$ is constant.

(ii) $f(z)=(a z+b)^{d_{1}}$ and $g(z)=c(a z+b)^{d_{2}}$, with $a, b, c$ constants and $d_{1}, d_{2}$ negative integers.

(iii) $f(z)=e^{a z+b}$ and $g(z)=e^{c z+d}$ with $a, b, c, d$ constants.

(iv) $f(z)=d_{1}\left(e^{Q_{1}}-d_{2}\right)^{-1}$ and $g(z)=d_{3}\left(1-d_{2} e^{-Q_{1}}\right)^{-1}$, with the $d_{3}$ constants and with $Q_{1}$ a non-constant polynomial.

(v) $f(z)=d_{1}\left(e^{d_{2} z}-d_{3}\right)$ and $g(z)=d_{4}\left(1-d_{3} e^{-d_{2} z}\right)$, with the $d_{3}$ constants.

(vi) $g(z)=d_{1}\left(e^{d_{2} z}+d_{3}\right) /\left(e^{d_{2} z}+3 d_{3}\right)$ and $f(z)=d_{4}\left(e^{d_{2} z}+d_{3}\right) /\left(e^{d_{2} z}+3 d_{3}\right)^{3}$, with the $d$, constants.

Of course, $f$ and $g$ may be interchanged in (vi), and it is easy to verify that $f$ and $g$ as given in (vi) do satisfy the hypotheses of Theorem 2. Further, simple examples such as $f(z)=\left(1-e^{z}\right)^{-1}$ and $g(z)=\left(1-e^{z}\right)^{-2}$ show that the hypo- 
thesis on $f^{\prime \prime}$ and $g^{\prime \prime}$ in Theorem 2 cannot be deleted. The proof of Theorem 2 uses a combination of Theorem 1 and asymptotic methods which do not readily extend to the infinite order case. In any case, Theorem 2 as stated would not be true for infinite order, as the following example, one of several from $[2,8]$, shows: set $f(z)=\exp \left(e^{z}-z\right), g(z)=\exp \left(e^{-z}+z\right)$.

\section{Proof of Theorem 1}

Given $f$ and $g$ as in the hypotheses, $f$ must be non-constant, since $g$ is. There exist polynomials $P$ and $Q$ and rational functions $R$ and $S$ such that

$$
f=R e^{P} g, \quad f^{\prime}=R e^{P} S e^{Q} g^{\prime} .
$$

If $S e^{Q} \equiv 1$ then (2.1) implies that $f^{\prime} / f \equiv g^{\prime} / g$ so that $f / g$ is constant. We assume henceforth that $f / g$ is non-constant. Now (2.1) gives

$$
f^{\prime}=\left(R^{\prime} / R+P^{\prime}\right) R e^{P} g+R e^{P} g^{\prime}, \quad g^{\prime} / g=\frac{R^{\prime} / R+P^{\prime}}{S e^{Q}-1} .
$$

If $Q$ is constant then (2.2) implies that $g^{\prime} / g$ is rational and so is $f^{\prime} / f$, by (2.1). We assume henceforth that $Q$ is non-constant, of degree $m \geqq 1$. We choose a real $\phi$ such that $\arg Q(z) \rightarrow \pi / 2$ as $z \rightarrow \infty$ with $\arg z=\phi$, and we write $L(z)=$ $Q(z)+\log S(z)$ and $H(z)=L(z)^{1 / m}$. There exists a non-zero constant $d$ such that $H^{\prime}(z)=d+O(1 /|z|)$ in the region $D$ given by $|\arg z-\phi|<2 \pi / 3 m,|z|>c_{1}$, using $c_{j}$ to denote constants. Now, any two distinct points $z_{1}, z_{2}$ in $D$ can be joined by a path through $D$ of length $O\left(\left|z_{1}-z_{2}\right|\right)$ and, integrating along this path

$$
\left|\int_{z_{1}}^{z_{2}}\left(H^{\prime}(z)-d\right) d z\right| \leqq \int_{z_{1}}^{z_{2}} O(1 /|z|)|d z| \leqq O\left(\left|z_{1}-z_{2}\right| c_{1}^{-1}\right) .
$$

Consequently, $H\left(z_{1}\right)-H\left(z_{2}\right)=\left(z_{1}-z_{2}\right)\left(d+O\left(1 / c_{1}\right)\right) \neq 0$ provided $c_{1}$ is large enough. Thus $w=H(z)=d z(1+o(1))$ is univalent on $D$, mapping into a sectorial region on which the mapping $w \rightarrow w^{m}$ is univalent. We conclude that $L$ maps a subdomain $D_{1}$ of $D$ univalently onto a half-plane $D_{2}$ given by $\operatorname{Im}(L)>c_{2}$. Points in $D_{2}$ with $L / 2 \pi i$ a positive integer correspond to poles of $g^{\prime} / g$ in $D_{1}$, these poles having residue $\left(R^{\prime} / R+P^{\prime}\right) /\left(S^{\prime} / S+Q^{\prime}\right)$. It follows from Lemma $A$ that there must be a polynomial $P_{1}$ such that

$$
R^{\prime} / R+P^{\prime}=P_{1}(L)\left(S^{\prime} / S+Q^{\prime}\right) .
$$

Since $L=Q+\log S$, the relation (2.3) can only hold if either $P_{1}$ is a constant, or $S$ is a constant. If $P_{1}$ is a constant $c$ then we obtain

$$
g^{\prime} / g=c\left(S^{\prime} / S+Q^{\prime}\right) \frac{S^{-1} e^{-Q}}{1-S^{-1} e^{-Q}} .
$$

Solving for $g$ and using the fact that $R e^{P}=c_{3}\left(S e^{Q}\right)^{c}$ we obtain 


$$
g=c_{4}\left(1-S^{-1} e^{-Q}\right)^{c}, \quad f=c_{5}\left(S e^{Q}-1\right)^{c},
$$

so that conclusion (c) holds. Now suppose that $P_{1}$ is non-constant. Then $S$ is constant, without loss of generality identically 1 . Then $R$ must also be constant, by (2.3), and $P^{\prime}=P_{1}(Q) Q^{\prime}$ and, using (2.1),

$$
g^{\prime} / g=\frac{P_{1}(Q) Q^{\prime}}{e^{Q}-1}, \quad f^{\prime} / f=\frac{P_{1}(Q) Q^{\prime}}{1-e^{-Q}},
$$

which is conclusion (d). We remark that in both cases (2.4) and (2.5), $f$ and $g$ must have either infinitely many poles or infinitely many zeros, and that in case (2.5) the multiplicities of these zeros or poles tend to infinity. This completes the proof of part (i).

We now prove part (ii), and thus assume that $f^{\prime \prime} / g^{\prime \prime}$ has only finitely many zeros and poles. The first possibility is that $f^{\prime} / g^{\prime}$ is constant, from which, using (2.1), it is easy to solve for $f$ and $g$, to obtain (c), with $d_{2}=-1$. The second possibility is that $f^{\prime \prime} / f^{\prime}$ and $g^{\prime \prime} / g^{\prime}$ are both rational, in which case the remark in the previous paragraph implies that either $f^{\prime} / f$ and $g^{\prime} / g$ must both be rational, or we have (2.4), with $c=1$, and conclusion (c) again.

On the other hand, we may have representations corresponding to (2.4) or (2.5) for $f^{\prime}$ and $g^{\prime}$. To determine $f$ and $g$ in this last case we first suppose that (2.4) holds. If $c \neq 1$ we obtain

$$
f^{\prime}=c_{6}\left(S^{\prime} / S+Q^{\prime}\right) S e^{Q}\left(S e^{Q}-1\right)^{c-1},
$$

which implies that $f^{\prime}$ has infinitely many zeros or poles of fixed multiplicity $|c-1|$. Since we are assuming a representation analogous to (2.4) or (2.5) for $f^{\prime}$ we must have

$$
f^{\prime}=c_{7}\left(T e^{U}-1\right)^{c_{8}}
$$

with $T$ rational and $U$ a non-constant polynomial. Clearly, $c_{8}=c-1$. However, we now see that the representations (2.6) and (2.7) are incompatible, since (2.7) implies that there are sectors in which $f^{\prime}(z)= \pm c_{7}(1+o(1))$ and this contradicts (2.6). Thus $c$ must be 1 if (2.4) holds, and once again we have conclusion (c).

Finally, we suppose that (2.5) holds, and that we have a representation corresponding to (2.4) or (2.5) for $f^{\prime}$. Then $f$ has either poles or zeros of large multiplicities, and this forces a representation

$$
f^{\prime \prime} / f^{\prime}=\frac{V}{e^{W}-1}
$$

in which $V$ and $W$ are non-constant polynomials. However, (2.5) implies that in sectors where $e^{Q}$ is large we have $f^{\prime}(z) / f(z)=P^{\prime}(z)+O\left(|z|^{-\mu}\right)$, and here it is possible to choose $\mu$ arbitrarily large. This gives $f^{\prime \prime}(z) / f(z)=P^{\prime \prime}(z)+P^{\prime}(z)^{2}+$ $O\left(|z|^{-\mu / 2}\right)$ and $f^{\prime \prime}(z) / f^{\prime}(z)=P^{\prime \prime}(z) / P^{\prime}(z)+P^{\prime}(z)+O\left(|z|^{-\mu / 4}\right)$, possibly in a slightly narrower sector. Since $V$ is a polynomial in (2.8), this can only occur if $P^{\prime}$ is constant. But $P^{\prime}(z)=P_{1}(Q(z)) Q^{\prime}(z)$ and $P_{1}$ and $Q$ are, by assumption, non-con- 
stant. This is a contradiction, so that (2.5) is impossible here, and Theorem 1 is proved.

\section{Proof of Theorem 2}

Suppose that $f$ and $g$ satisfy the hypotheses of Theorem 2 . We can assume that $f / g$ is non-constant, since otherwise there is nothing to prove. If $f$ is constant then so is $g$, and we assume henceforth that $f$ and $g$ are both nonconstant. Then each pole of $f^{\prime} / f$ is a zero or pole of $f$ and so a simple pole of $g^{\prime} / g$. Further, each zero of $f^{\prime} / f$ is a zero of $f^{\prime}$ which is not a zero of $f$, and hence a zero of $g^{\prime} / g$. Applying the same reasoning to $f^{\prime}$ and $g^{\prime}$, we see that there are polynomials $Q$ and $S$ such that

$$
f^{\prime} / f=e^{Q} g^{\prime} / g, \quad f^{\prime \prime} / f^{\prime}=e^{S} g^{\prime \prime} / g^{\prime} .
$$

In addition we write

$$
H=g / g^{\prime}=e^{Q} f / f^{\prime}, \quad H^{\prime}\left(1-e^{S}\right)=Q^{\prime} H+e^{Q}-e^{S} .
$$

Suppose first of all that all but finitely many poles of $f$ and $g$ are such that the multiplicities corresponding to $f$ and $g$ coincide. Then we may apply Theorem 1, and we have either conclusion (b) or conclusion (c) of that theorem. In the first case $e^{Q}$ and $e^{S}$ must both be constant and, by (3.2), either $e^{S}=e^{Q}=1$, in which case $f / g$ is constant, or $H^{\prime}$ is constant and we obtain (i), (ii) or (iii). On the other hand, if conclusion (c) of Theorem 1 holds, we can write $f(z)=$ $d_{1}\left(S_{1} e^{Q_{1}}-1\right)^{d_{2}}$, with $d_{1}$ constant, $d_{2}= \pm 1$, and with $S_{1}$ rational and $Q_{1}$ a nonconstant polynomial. If $d_{2}=-1$ then $f^{\prime} / g^{\prime}$ is constant, so that $e^{S}=1$ and (3.2) gives $g^{\prime} / g=Q^{\prime} /\left(1-e^{Q}\right)$. Thus we have conclusion (iv). Finally, if $d_{2}=1$, we can write, in a sectorial region, $f=d_{1}\left(e^{T_{1}}-1\right)$ and $g=D_{1}\left(1-e^{-T_{1}}\right)$ with $D_{1}$ a constant and $T_{1}=Q_{1}+\log S_{1}$. This gives

$$
f^{\prime \prime} / f^{\prime}=T_{1}^{\prime \prime} / T_{1}{ }^{\prime}+T_{1}{ }^{\prime}, \quad g^{\prime \prime} / g^{\prime}=T_{1}^{\prime \prime} / T_{1}{ }^{\prime}-T_{1}{ }^{\prime},
$$

which is only compatible with (3.1) if $T_{1}{ }^{\prime}$ is constant, and this leads to (v). We assume henceforth that $f$ and $g$ have infinitely many poles at which the multiplicities corresponding to $f$ and $g$ do not coincide.

Since $f$ and $g$ have finite order, there exist positive constants $M_{1}, M_{2}$ and a set $V$, which is the union of $\operatorname{discs} D_{\jmath}=B\left(z_{\jmath},\left|z_{j}\right|^{-M_{1}}\right)$, where $z_{j} \rightarrow \infty$ as $j \rightarrow \infty$, such that for $|z|$ large with $z$ lying outside $V$ we have, for $0 \leqq \jmath<k \leqq 2$, the estimate

$$
\left|f^{(k)}(z) / f^{(\jmath)}(z)\right|+\left|g^{(k)}(z) / g^{(j)}(z)\right| \leqq|z|^{M_{2}} .
$$

The set $V$ can be constructed from discs centred at zeros and poles of $f, f^{\prime}, g$ and $g^{\prime}$ and, by taking $M_{1}$ large enough, we can ensure that the sum of the radii of the discs of $V$ is finite. We note $[5$, p. 84$]$ that the set of $\theta \in[0,2 \pi]$ such that the ray $\arg z=\theta$ meets infinitely many of the discs of $V$ has measure 
0. We now consider two cases :

CASE 1: Suppose that $Q$ and $S$ are both non-constant.

We use the following notation [5]. If $Q$ has degree $m$, and leading term $\alpha^{*} z^{m}$, then we write, for $\theta$ real, $\delta(Q, \theta)=\operatorname{Re}\left(\alpha^{*}\right) \cos (m \theta)-\operatorname{Im}\left(\alpha^{*}\right) \sin (m \theta)$, which is positive or negative according to whether $e^{Q}$ is large or small on $\arg z=\theta$.

Proceeding with the proof, we first note that the degrees of $Q$ and $S$ must be the same. To prove this, we assume that $Q$ and $S$ have different degrees, and it is then easy to see that there exists a real number $\phi$ such that the ray $\arg z=\phi$ meets at most finitely many of the discs of $V$ and such that $\delta(Q, \phi)$ $<0$ and $\delta(S, \phi)<0$. From (3.1) and (3.3) we see that if $s$ is large then

$$
\int_{s}^{+\infty}\left|f^{\prime \prime}\left(t e^{\imath \phi}\right) / f^{\prime}\left(t e^{\imath \phi}\right)\right| d t \leqq s^{-2}
$$

and this implies the existence of a non-zero constant $c_{0}$ such that $f^{\prime}(z)=c_{0}+$ $O\left(|z|^{-2}\right)$ on the ray $\arg z=\phi$. A further integration yields, for some constant $d_{0}$, an estimate $f(z)=c_{0} z+d_{0}+O\left(|z|^{-1}\right)$ on $\arg z=\phi$, so that we have, on this same ray, $f^{\prime}(z) / f(z)=(1+o(1)) / z$. But this, on combination with (3.1) and (3.3) and the fact that $\delta(Q, \phi)<0$, yields a contradiction, proving our assertion.

We can now write

$$
S(z)=C Q(z)+P(z),
$$

in which $C$ is a non-zero constant and the degree of $P$ is strictly less than the degree of $Q$, and we assert:

$$
C \text { is real and negative. }
$$

If $C$ is not real, then the rays $\arg z=\theta$ with $\delta(Q, \theta)=0$ are simply the rays $\arg z=\phi$ with $\delta(S, \phi)=0$ rotated through an angle $\alpha_{1} \in(0, \pi / m)$. Therefore, if (3.5) does not hold, we can again find a ray $\arg z=\phi$ with $\delta(Q, \phi)$ and $\delta(S, \phi)$ both negative, such that this ray meets only finitely many of the discs of the $R$-set $V$. As before, we obtain a contradiction, and (3.5) is proved.

We now set

$$
F=\frac{e^{Q}-1}{f},
$$

and note that by (3.1) poles of $F$ arise only from multiple zeros of $f$. Again by (3.1), at a pole $z_{1}$ of $f$ of multiplicity $n_{1}$, at which $g$ has a pole of multiplicity $m_{1}$, we have

$$
e^{Q-S}=n_{1}\left(m_{1}+1\right) / m_{1}\left(n_{1}+1\right),
$$

the right-hand side being bounded above and below in absolute value. This implies that if $\left|z_{1}\right|$ is large then $\arg z_{1}=\theta^{*}+o(1)$ for some $\theta^{*}$ with $\delta\left(Q-S, \theta^{*}\right)$ 
$=\delta\left((1-C) Q-P, \theta^{*}\right)=0$. Further, zeros of $f$ can only occur where $e^{Q}=1$, and zeros of $f^{\prime}$ where $e^{S}=1$, and the rays $\arg z=\theta^{*}$ with $\delta\left(Q, \theta^{*}\right)=0, \delta\left(S, \theta^{*}\right)=0$, $\delta\left(Q-S, \theta^{*}\right)=0$ coincide, using the fact that $C<0$. Let $m$ be the degree of $Q$ and let $\theta_{k}, 1 \leqq k \leqq 2 m+1$, with $\theta_{k+1}=\theta_{k}+\pi / m$, be such that $\delta\left(Q, \theta_{k}\right)=0$. Further, let $\varepsilon$ be small and positive. The estimate (3.3) holds for all $z$ with $|z|$ large and

$$
\arg z=\theta, \quad \theta_{k}+\varepsilon \leqq \theta \leqq \theta_{k+1}-\varepsilon .
$$

Suppose first that $\delta(Q, \theta)<0$ on the interval (3.7), so that $\delta(S, \theta)>0$. Then (3.1) and (3.3) imply that $g^{\prime \prime}(z) / g^{\prime}(z)=O\left(|z|^{-4}\right)$ in the corresponding sectorial region, giving $g^{\prime}(z)=c_{1}+O\left(|z|^{-3}\right)$ and $g(z)=c_{1} z+c_{2}+O\left(|z|^{-2}\right)$, with the $c_{\text {, con- }}$ stants and $c_{1} \neq 0$. Further, $f^{\prime}(z) / f(z)=e^{Q(z)} z^{-1}(1+o(1))$, which gives $f(z)=c_{3}+o(1)$, with $c_{3}$ a non-zero constant. (3.6) gives

$$
F^{\prime}(z)=e^{Q}\left(Q^{\prime}-f^{\prime} / f\right) / f+f^{\prime} / f^{2}=Q^{\prime}(z) e^{Q(z)}\left(c_{3}+o(1)\right)^{-1} .
$$

Suppose now that $\delta(Q, \theta)>0$ on the interval (3.7). Then we obtain $f^{\prime}(z)=d_{3}+$ $O\left(|z|^{-3}\right)$ and $f(z)=d_{3} z+d_{4}+O\left(|z|^{-2}\right)$, with the $d$, constants, $d_{3}$ not zero. Thus

$$
F^{\prime}(z)=e^{Q(z)}\left(Q^{\prime}(z)+O(1 /|z|)\right) / f+o(1)=Q^{\prime}(z) e^{Q(z)}\left(d_{3} z\right)^{-1}(1+o(1)) .
$$

Applying the Phragmén-Lindelöf principle to the function $F^{\prime}(z) Q^{\prime}(z)^{-1} e^{-Q(z)}$, which has finite order, we deduce that, for each $k$, and for each $\varepsilon>0$, the function $F^{\prime}$ has infinitely many poles, and hence $f$ has infinitely many multiple zeros, in the sectorial region $S_{k, \varepsilon}$ given by $|z|$ large, $\left|\arg z-\theta_{k}\right|<\varepsilon$. We can now assert:

$P$ is constant.

If (3.10) is false, we can find $\theta_{k}$ and $\varepsilon>0$ such that $e^{P \rightarrow 0}$ as $z \rightarrow \infty$ in $S_{k, \varepsilon}$. This region contains infinitely many multiple zeros of $f$, at which $e^{Q}=e^{S}=1$, which gives, using (3.4), $1=\left|e^{C Q+P}\right|=\left|e^{P}\right| \rightarrow 0$, a contradiction.

We now have, using (3.1) and (3.4),

$$
\left(f^{\prime} / f\right)^{-C} f^{\prime \prime} / f^{\prime} \equiv A\left(g^{\prime} / g\right)^{-C} g^{\prime \prime} / g^{\prime}
$$

for some non-zero constant $A$. At a multiple zero of $f$ we must have $e^{Q}=1$ and $A e^{C Q}=1$. Writing $Q=2 \pi i Q_{2}$ and $A=e^{2 \pi i B}$ we therefore have infinitely many integers $n_{1}$ such that $C n_{1}+B$ is an integer, from which it follows at once that $C$ and $B$ are real and rational, and we can write

$$
-C=p_{1} / s_{1}, \quad A=e^{2 \pi \imath q_{1} / s_{1}},
$$

with $p_{1}, q_{1}, s_{1}$ integers, $p_{1}, s_{1}$ positive. Suppose now that $f$ has a pole of order $n_{1}$, and $g$ a pole of order $m_{1}$ at some point $z_{1}$. Then we have

$$
\left(-n_{1}\right)^{p_{1}}\left(-n_{1}-1\right)^{s_{1}}=\left(-m_{1}\right)^{p_{1}}\left(-m_{1}-1\right)^{s_{1}}
$$


from which it follows that $m_{1}=n_{1}$, so that $f$ and $g$ share $\infty \mathbf{C M}$, and we have already disposed of this case. This completes our discussion of Case 1.

CASE 2: Suppose that $Q$ or $S$ is constant.

If $e^{Q}$ is a constant $c \neq 1$, then we have, by (3.2), $H^{\prime}\left(1-e^{S}\right)=c-e^{S}$, which forces $S$ to be constant, for otherwise $H^{\prime}$ would have to have simple poles. This makes $H=g / g^{\prime}$ linear, and leads to (ii) or (iii).

Now suppose that $e^{S}$ is a constant $c$. If $c=1$ then $f^{\prime} / g^{\prime}$ is constant and $f=a g+b$, with $a, b$ constants. This means that $f$ and $g$ share $\infty \mathbf{C M}$ and we have dealt with this case. We assume henceforth that $c \neq 1$, and may suppose that $c$ is a positive rational number, since otherwise $f$ and $g$ cannot have poles. Further, we can assume that $c>1$, interchanging $f$ and $g$ and $Q$ and $-Q$ otherwise, and we recall that, by assumption, $g$ has infinitely many poles. Now (3.2) leads to

$$
g^{\prime} / g=\frac{(1-c) e^{\beta Q}}{A_{0}+\int_{0}^{z} e^{\alpha Q}-c e^{\beta Q} d t}, \quad A_{0} \in \boldsymbol{C}, \quad \beta=1 /(c-1), \quad \alpha=1+\beta=c \beta .
$$

We assume for the time being that $Q$ is non-linear, of degree $m \geqq 2$. Since $g$ has infinitely many poles, we can choose a real $\phi$ such that $\delta(Q, \phi)=0$ and such that $g$ has infinitely many poles in $|\arg z-\phi| \leqq \pi / 2 m$. Repeated integration by parts shows that, in $|\arg z-\phi|<(\pi / m)-\varepsilon$, the denominator of (3.11) is

$$
u(z)=A_{1}+Q^{\prime}(z)^{-1} e^{\beta Q(z)}\left[e^{Q(z)}(1+o(1)) \alpha^{-1}-c(1+o(1)) \beta^{-1}\right], \quad A_{1} \in \boldsymbol{C} .
$$

We can take a pole $z$ of $f$ and $g$, the multiplicites being $n_{1}$ and $m_{1}$ respectively, and with $|z|$ large, and we must have $u(z)=0$ and $e^{Q(z)}=n_{1} / m_{1}$, and $\left(n_{1}+1\right) /$ $\left(m_{1}+1\right)=c$, the last equation implying that $|\log | n_{1} / m_{1}|| \leqq O(1)$. This gives us $0=u(z)=A_{1}+o(1)$, so that $A_{1}$ must be zero. Therefore, $n / m_{1}=(1+o(1)) c \alpha / \beta=$ $(1+o(1)) c^{2}$. If $n_{1}$ takes arbitrarily large values here, we obtain $c^{2} \sim n_{1} / m_{1} \sim$ $\left(n_{1}+1\right) /\left(m_{1}+1\right)=c$, forcing $c=1$, which we have assumed to not be the case. Therefore $n_{1}$ and $m_{1}$ are both bounded, and in the relation $c^{2} \sim n_{1} / m_{1}$ there are only finitely many values of $n_{1} / m_{1}$ which can occur. Therefore, if $z$ is large enough we must in fact have $n_{1} / m_{1}=c^{2},\left(n_{1}+1\right) /\left(m_{1}+1\right)=c$, leading to $n_{1}=c$, $m_{1}=1 / c$, and again to $c=1$. This is a contradiction and we conclude that $Q$ must be linear.

We can therefore write

$$
\beta=p / q, \quad \alpha=(p+q) / q, \quad T=Q / q, \quad X=e^{T},
$$

with $p$ and $q$ positive integers, with greatest common divisor $(p, q)=1$, and

$$
g^{\prime} / g=(1-c) e^{p T}\left(A_{1}-c e^{p T} / \beta Q^{\prime}+e^{(p+q) T} / \alpha Q^{\prime}\right)^{-1} .
$$

Poles of $g^{\prime} / g$ occur where $K(X)=0$, the polynomial $K$ being defined by 


$$
K(X)=A_{2}-c^{2} X^{p}+X^{p+q}, \quad A_{2}=\alpha Q^{\prime} A_{1} .
$$

We assume for now that $A_{2} \neq 0$. Then the polynomial $K(X)$ has $p+q$ roots, all non-zero, say $X_{1}, \cdots, X_{p+q}$, which must be distinct, since $g^{\prime} / g$ has only simple poles. Now, at a zero of $g$ we have $X^{q}=e^{Q}=1$, and $\arg \left(X^{p}\right)=\arg \left(A_{2} /\left(c^{2}-1\right)\right)$ $(\bmod 2 \pi)$, this relation being satisfied by at most one $q^{\prime}$ th root of unity, since $(p, q)=1$. Therefore, at most one of the roots $X$, can give rise to zeros of $g$. Further, at a zero of $g$ we have $H=0$ and, by (3.2), $H^{\prime}=1$, and so all zeros of $g$ are simple. But we can easily estimate the number of zeros of $\mathrm{e}^{T}-X_{j}$ in $|z| \leqq r$, and we see now that

$$
\bar{n}(r, g) \geqq(p+q-1-o(1)) n(r, 1 / g), \quad r / \bar{n}(r, g)=O(1) .
$$

On the other hand, (3.12) implies that off a set of angular measure at most $\varepsilon$, we have $g^{\prime}(z) / g(z)=o\left(|z|^{-1}\right)$. Further, it is easy to see that there are arbitrarily large $r$ for which

$$
\max _{\jmath}\left|\mathrm{e}^{T^{(z)}}-X_{\jmath}\right|^{-1} \leqq O(1), \quad|z|=r .
$$

A partial fractions decomposition of the right-hand side of (3.12), viewed as a rational function in $e^{T}$, now shows that $n(r, 1 / g)-n(r, g)=o(r)$ for these $r$, contradicting (3.13), unless $p=q=1$.

If $p=q=1$ (and $A_{2}$ is non-zero), the argument of the previous paragraph shows that $g$ must have the form given in conclusion (vi) of the theorem. Further, $\beta=1$ so that $c=2$ and (3.1) implies that $f^{\prime}$ is a constant multiple of $\left(g^{\prime}\right)^{2}$. Determining $f^{\prime}$ from $g$ and using integration by parts and the fact that $f$ and $g$ share the value $0 \mathbf{C M}$, we obtain the asserted form for $f$.

The only remaining case to consider is when $A_{2}=0$. However, here (3.1) and (3.12) give $g^{\prime} / g=-c Q^{\prime}\left(e^{Q}-c^{2}\right)^{-1}$. Thus the residue of $g^{\prime} / g$ at each of its poles must be $-1 / c$, which contradicts our earlier assumption that $c>1$. The proof of Theorem 2 is complete.

The author would like to thank the referee for several helpful comments.

\section{REFERENCES}

[1] K. F. Barth, D. A. Brannan and W.K. HaYman, Research problems in complex analysis, Bull. London Math. Soc., 16 (1984), 490-517.

[2] G.G. Gundersen, When two entire functions and also their first derivatives have the same zeros, Indiana Univ. Math. J., 30 (1981), 293-303.

[3] W.K. Hayman, Meromorphic Functions, Clarendon Press, Oxford, 1964.

[4] L. Köhler, Meromorphe Funktionen mit gleichen Nullstellen und Polstellen und jeweils gleichen Nullstellen einiger Ableitungen, Doktordissertation, Hannover, 1987.

[5] L. KöHLER, Meromorphic functions sharing zeros and poles and also some of their derivatives sharing zeros, Complex Variables Theory Appl., 11 (1989), $39-48$. 
TWO RESULTS RELATED TO A QUESTION OF HINKKANEN

[6] I. LAINE, Nevanlinna theory and complex differential equations, de Gruyter Stud. Math., 15, Walter de Gruyter, Berlin-New York, 1993.

[7] J.K. LANGLEY, On second order linear differential polynomials, Results Math., 26 (1994), 51-82.

[8] K. ToHge, On a problem of Hinkkanen about Hadamard products, Kodai Math. J., 13 (1990), 101-120.

[9] J.M. WhitTAKeR, Interpolatory Function Theory, Cambridge Tract in Math., 33, Cambridge University Press, 1935.

DepaRTMENT OF MATHEMATICS

UNIVERSITY OF NOTTINGHAM

NG7 2RD, UK

e-mail: jkl@maths.nott.ac.uk. 